\title{
La contaminación ambiental ocasionada por la minería en la provincia de El Oro
}

\author{
Environmental Pollution as a Result \\ of Mining in the Province of El Oro
}

A contaminação ambiental ocasionada pela mineração na província El Oro

\author{
Wilson Vilela-Pincay \\ Universidad Técnica de Machala. Machala, Ecuador \\ wvilela@utmachala.edu.ec \\ https://orcid.org/0000-0002-0786-7622

\section{Marbelle Espinosa-Encarnación} \\ Universidad Técnica de Machala. Machala, Ecuador \\ mespinosa3@utmachala.edu.ec \\ https://orcid.org/0000-0001-6524-6384

\section{Ana Bravo-González} \\ Universidad Técnica de Machala. Machala, Ecuador \\ abravo2@utmachala.edu.ec \\ https://orcid.org/0000-0003-0727-9000
}

DOI: https://doi.org/10.32719/25506641.2020.8.8

Recibido: 18 de febrero de 2020 - Revisado: 4 de marzo de 2020

Aceptado: 12 de mayo de 2020

Artículo de investigación

Licencia Creative Commons

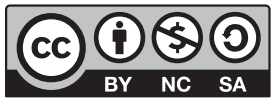




\section{Resumen}

La contaminación ambiental ocasionada por la minería en la provincia de El Oro ha traído consecuencias graves e irreparables al medioambiente; la minería ha estado presente ya desde tiempo atrás provocando un descontrol realizado por las actividades mineras en los cantones de la parte alta de la provincia. El objetivo de esta investigación es develar el nivel de participación social en el cuidado y preservación por la naturaleza, para lo cual se precisó como metodología el empleo de una investigación descriptiva y cualitativa en la que se estableció de la manera más completa posible la explicación a fondo del fenómeno y la problemática, con el fin de resultados a través de la observación. El diseño de investigación cualitativo escogido para el presente trabajo ha permitido establecer una diferencia entre los datos recopilados por las fuentes bibliográficas y los resultados obtenidos por los investigadores a través de la observación, para finalmente concluir que la minería es considerada como una de las actividades más perjudiciales para el medioambiente, por sus consecuencias y efectos que deja; sin embargo, esto no significa que se deba terminar de raíz con la minería. Se debería llegar a una explotación de minerales que no traiga consecuencias negativas al medioambiente, porque los principales perjudicados seremos nosotros.

Palabras clave: Minería, población, impacto ambiental, minerales, ambiente.

JEL: Q53 Contaminación del aire; contaminación del agua; ruido; residuos peligrosos; reciclaje de residuos sólidos.

\section{Summary}

The environmental pollution caused by mining in the Province of El Oro has brought serious and irreparable consequences to the environment. Mining has been there for a long time, causing a great damage by its activities in the cantons located in the upper part of the province. The objective of this research is to unveil the level of social participation in the care and preservation of nature, for this purpose the use of descriptive and qualitative research was required as a methodology in which the explanation on the background of the phenomenon and the problems was established obtaining the results through observation. The qualitative research design chosen for this work has allowed us to establish a difference between the data collected by bibliographic sources and the results obtained by researchers through observation, to finally conclude that mining is considered one of the most detrimental activities against the environment, due to the consequences and effects mining leaves on it. However, this does not mean that mining must be completely eradicated, the exploitation of minerals should not bring negative consequences to the environment because the main victims will be us.

Keywords: Mining, population, environmental impact, minerals, environment.

JEL: Q53 Air pollution; water pollution; noise; hazardous waste; solid waste; recycling. 


\section{Resumo}

A contaminação ambiental ocasionada pela mineração na província El Oro, no Equador, tem trazido consequências graves e irreparáveis ao meio ambiente. A mineração tem estado presente há tempos, provocando um descontrole pelas atividades operadas na parte alta da província. O objetivo desta pesquisa é evidenciar o nível de participação social no cuidado e na preservação da natureza. Para tanto, utilizou-se como metodologia uma pesquisa descritiva e qualitativa na qual se estabeleceu, da maneira mais completa possível, a explicação aprofundada do fenômeno e de sua problemática, por meio da qual os resultados foram obtidos utilizando-se a observação. O modelo de pesquisa qualitativo escolhido para o presente trabalhou permitiu traçar uma diferença entre os dados coletados pelas fontes bibliográficas e os resultados obtidos pelos pesquisadores através da observação. Concluiu-se que a mineração é considerada uma das atividades mais prejudiciais para o meio ambiente devido às consequências e efeitos que deixa sobre o mesmo. No entanto, isso não significa que a mineração deva ser abolida, a exploração de minerais não deve trazer consequências negativas para o meio ambiente, pois os principais prejudicados seremos nós.

Palavras-chave: Mineração, população, impacto ambiental, minerais, ambiente.

JEL: Q53 Poluição do ar; poluição da água; ruído; resíduos perigosos; resíduos sólidos; reciclagem.

\section{Introduccion y estado de la cuestión}

$\mathrm{L}$

a actividad minera desarrollada en la provincia de El Oro ha ocasionado daños e impactos ambientales a la naturaleza y al ecosistema de los cantones de la parte alta de la provincia, reduciendo todo tipo de probabilidades de conservar el medioambiente sano. Según Henry y Heinke (1999, 568), "las soluciones dependen en gran medida del clima, la topografía y la geología y de la naturaleza de la operación de explotación minera"; además, esta problemática tiene muchos años de no ser atentida por las autoridades correspondientes y los mismos habitantes se encuentran acostumbrados, por lo que es necesario cambiar esa realidad.

El presente trabajo tiene como finalidad exponer la situación actual de Zaruma y Portovelo con respecto a la minería ilegal y develar el nivel de participación social en el cuidado de la contaminación ambiental y su preservación por la naturaleza. Según Alcívar $(2015,8)$, "la actividad minera en cantones como Zaruma y Portovelo se ha desarrollado de manera tradicional con la extracción del oro con tratamientos a base de cianuro y mercurio", lle- 
gando a formar parte del 19,45\% de la contaminación al agua en la provincia de El Oro.

\section{Marco teórico}

El derecho es una ciencia que "se basa en lo que podemos ver, oír, tocar y apreciar por los sentidos" (Bouzas et al. 2007, 22), y está sujeta a frecuentes opiniones y referencias personales de quienes la ejercen; por ende, el derecho ambiental necesita la atención del sistema jurídico, con normas que permitan la protección de la naturaleza y el medioambiente.

Según el Diccionario de la lengua española, la minería "es el arte de laborear las minas por medio de individuos que se dedican a este trabajo"; sin embargo, se puede decir que la minería es la obtención física selectiva de minerales y otros materiales no minerales que ofrece la corteza terrestre, haciendo referencia a los minerales como sustancia de origen natural, con una composición química definida y unas propiedades predecibles y constantes.

\section{Explotación minera}

No existe la posibilidad de pensar en calidad de vida y, consecuentemente, en desarrollo económico, sin la amplia utilización de recursos minerales $\mathrm{y}$, por tanto, sin la minería, de tal manera que el desarrollo del sector minero en un país, con una administración responsable, sería la herramienta principal para alcanzar una mejora, tanto en la calidad de vida como en el bienestar económico de la sociedad. Sin embargo, existe otro escenario en el que no se toman las medidas necesarias para proteger el medioambiente, ocasionando como resultado únicamente contaminación y destrucción.

Pero, ¿a qué se debe este impacto negativo? Según Alvarado $(2017,4)$, "en la actualidad, los riesgos se originan como consecuencia del desarrollo productivo, generando así un incremento en las circunstancias de peligro". Significa que la producción o la realización de actividades mineras no son negativas desde una perspectiva económica, pero, cuando un Estado se enfoca únicamente en la producción, queda de lado la protección de los derechos de la naturaleza, poniendo en riesgo el sistema ambiental. 
Para ello, el Código Orgánico Integral Penal EC (2014), en el artículo 260, sanciona con pena privativa de libertad, de siete a diez años, cuando las actividades mineras ilegales realizadas en el país ocasionan daño al medioambiente, de esta forma existe una garantía para la protección de los recursos naturales.

En países en vías de desarrollo como Ecuador es mas notorio evidenciar este tipo de situaciones, en las que el Estado ha aprovechado únicamente los recrusos naturales sin dar la debida protección a la naturaleza. Según Vargas y Manrique $(2015,3)$, "se ha encontrado que países en vía de desarrollo y pobres no lo son por su carencia de recursos", sino por su mala utilización, invirtiendo en proyectos que no resultan beneficiosos para los ciudadanos.

Para Sánchez, Espinosa y Eguiguren (2016, 3), las consecuencias de la actividad minera desembocan en problemas ambientales, y esos "problemas ambientales pueden constituirse en factores desencadenantes de muchos de los conflictos sociales, pero en sí mismos no constituyen un conflicto". La irresponsabilidad con la que se manejan los desechos provenientes de las minas es un conflicto social que necesita ser solucionado.

Según Sánchez, Lelfsen y Versú $(2017,9)$, “el sistema de propiedad colectiva de la tierra desempeña también un papel importante en la forma que toma el conflicto a partir de la llegada de la empresa minera". Para muchas comunidades y pueblos indígenas, el Sumak Kawsay, o buen vivir, es pura palabrería del gobierno del expresidente Rafael Correa en comparación con lo que realmente sucede al explotar la tierra.

Tanto fue en su momento el auge de la explotación minera, que en varios países de Europa se evidenció que dicha actividad "se convirtió en uno de los principales suministradores de materia prima, fundamentalmente minerales metálicos, a la industria europea" (Cueto 2016, 3). Con ello, la producción industrial aumentó, generando más ingresos económicos al país, y esta misma explotación minera a su vez promueve el turismo y el empleo.

El ser humano, a través de su paso por el planeta Tierra, ha realizado diversas actividades para sobrevivir, una de ellas, la explotación minera, convirtiéndola en una de sus principales fuentes de ingresos económicos. Sin embargo, no todo es beneficioso. Existe gran cantidad de contaminación proveniente de su entorno. Según Español (2012, 2), "la actividad del hombre ha generalizado los casos de exposición, contribuyendo con un legado 
de mercurio en vertederos, los desechos de la minería y los emplazamientos, suelos y sedimentos industriales contaminados".

La contaminación ambiental es el resultado de avances tecnológicos y la imparable globalización. Bouzas $(2015,203)$ manifiesta que "la globalización alude a un conjunto de procesos económicos, políticos, socioculturales, científico-tecnológicos, ambientales y geográficos sumamente complejos y cambiantes". Es importante que absolutamente todas las personas, incluidos los pequeños empresarios y las grandes empresas, sean amigables con el medioambiente y realicen sus respectivos protocolos para la conservación y preservación de la naturaleza, no para evitar las posibles sanciones que implica contaminar, sino por alargar el tiempo de vida de cada ser vivo en la tierra.

\section{Situación de la actividad minera en la provincia de El Oro: Zaruma y Portovelo}

La Secretaría de Gestión de Riesgos hizo una radiografía del subsuelo de Zaruma, principal cantón afectado por la actividad minera en la provincia de El Oro, para detectar posibles zonas de riesgo de hundimientos por minería. Marcelo Cando, subsecretario de Información de Gestión de Riesgos, explica que ya tienen datos preliminares de lo que sucedió en La Inmaculada.

Según González et al. $(2018,2)$, "los metales considerados más tóxicos y de riesgo para el ambiente son el cadmio, mercurio y plomo", algunos de ellos provenientes de las minas. Por otro lado, Castro $(2019,25)$ manifiesta que "el minero va al río a lavar el oro encontrado, y en el agua deja residuos de plomo, mercurio, manganesio y cianuro, dejándolo completamente contaminado y por tanto su agua no apta para el consumo humano".

La Agencia de Regulación y Control Minero (ARCOM) registra 92 concesiones mineras entre Zaruma y Portovelo, su cantón vecino; la entidad además ha escaneado 65 kilómetros de galerías. Según Paucar (2017, párr. 10), para diario El Comercio, "una galería es una especie de túnel que sigue la veta", mientras que "una veta es un depósito de mineral (oro, plata o cobre), adherido a rocas".

Paucar (2017, párr. 25) también manifiesta que: 
Zaruma tiene 24.100 habitantes. El $40 \%$ vive en el área urbana, donde hay 3.200 predios. Esa es gran parte de la zona de exclusión, donde hasta hace unos meses seguían escuchando las detonaciones mineras. La historia de esta localidad está ligada al oro. Actualmente, el cantón reporta 45 concesiones mineras que emplean a 890 personas, en su mayoría de otras provincias y hasta de países cercanos.

\section{El impacto ambiental por la actividad minera en la región de Zaruma y Portovelo}

El Gobierno del Ecuador declaró el 14 de septiembre de 2017 el estado de excepción por sesenta días en la ciudad de Zaruma, afectada por la actividad minera que ha socavado el subsuelo de la urbe, declarada patrimonio cultural del país. Según López et al. (2016, 4), "al momento existen cientos de pequeñas minas de oro en la zona, que son inseguras, mal ventiladas y calurosas, con riesgos constantes de derrumbes", razón por la cual el Estado ecuatoriano ha puesto mano dura a la minería ilegal realizada en Zaruma.

Frecuentemente se presentan conflictos entre las estrategias para extraer los minerales de la tierra, "maximizando las ganancias a corto plazo (5 a 10 años) y aquellas que maximizan los beneficios a largo plazo (50 años)"; las primeras traen como consecuencia la irreversible degradación ambiental (Vázquez y Valdez 1994, 158).

Se puede resaltar que los recursos utilizados por las personas para llevar a cabo actividades como la minería tienen un sinnúmero de reacciones negativas para el medioambiente, pero, ¿qué es en realidad el impacto ambiental que generan este tipo de dinamismos para el ecosistema? Es decir, la labor minera ha traído consigo cambios exponenciales para el ambiente. Orellana, Briére y Rodríguez $(2020,17)$ expresan que "el impacto de la actividad industrial sobre los sistemas que sustentan la vida es de tal envergadura, que está poniendo en peligro la existencia de diversas formas de vida, incluyendo la supervivencia de la humanidad misma".

Esta amarga realidad experimenta una fuerte contaminación debido a la explotación de los recursos naturales no renovables, entre ellos los productos provenientes del subsuelo o los yacimientos minerales, concentrándose en lugares como la parte alta de la provincia, que son ricos para la extracción de metales, sin tener en cuenta los efectos negativos resultantes, tanto para la 
población como para el ambiente que los rodea. La Agencia de Regulación y Control Minero $(2015,10)$ apunta que "es considerado uno de los distritos mineros más importantes del país, se encuentra ubicado en la provincia de El Oro, comprende los cantones de Zaruma, Portovelo y Atahualpa".

Debido a la gran demanda de la actividad minera que se realizó en las ciudades de Zaruma y Portovelo, bajo suelo, literalmente, se empezaron a hundir, llevándose consigo hasta ahora escuelas y edificaciones por el mal manejo. Surgió entonces miedo en la población por su existencia. Con el pasar de los días, los estruendos en las ciudades fueron más frecuentes, como parte de las fases de prospección, exploración, explotación y fundición efectuadas en los subsuelos para la extracción de las áreas mineralizadas. En Ecuador, el Ministerio de Minería (2016) estableció que "La contaminación generada se da principalmente por la minería ilegal y de aquellas que no cuentan con las autorizaciones y licencias ambientales, mismas que al ser poco tecnificadas, y no cumplir con la normativa ambiental vigente, han afectado gravemente su medio".

Según Román (2020, párr. 2), en cartas hacia el director del periódico El Universo manifestó:

La comunidad no ha cesado de defender a Zaruma de la explotación de oro en su subsuelo urbano, ciudad patrimonio con muchos títulos de honor que no le han servido. La colonia de zarumeños residentes en Quito logró que la Comisión de la Biodiversidad de la Asamblea Nacional sesione en Zaruma el 15 de enero de 2020, pero no pudo hacerlo porque ocho de sus once integrantes no concurrieron a esta cita, solo enviaron a sus representantes, violando la disposición de su obligatoriedad de estar presentes. Por falta de quorum se limitaron a escuchar nuestras quejas. El Ministerio de Energía, el 12 de septiembre de 2019, expidió el Acuerdo Ministerial 2019-050 para continuar las actividades mineras bajo la ciudad de Zaruma.

Paucar (2017, párr. 8) expresa lo siguiente:

La Secretaría de Gestión de Riesgos hace una radiografía del subsuelo del cantón para detectar posibles zonas de riesgo de hundimientos por minería. Marcelo Cando, subsecretario de Información de Gestión de Riesgos, explica que ya tienen datos preliminares de lo que sucedió en La Inmaculada. "Por estudios geofísicos se ha determinado que hay unas cabezas de minas abajo, que posiblemente se hundieron y causaron el socavón”. 


\section{Efectos negativos: minería a cielo abierto}

La minería a cielo abierto utiliza, de acuerdo con Sánchez y Ortiz (2014, 1), "grandes cantidades de cianuro de manera intensiva", que le permiten recuperar el oro del resto del material removido. Para desarrollar este proceso se requiere que el yacimiento abarque grandes extensiones y que se encuentre cerca de la superficie. Además, se cavan cráteres grandes en extensión y en profundidad.

Según Pérez y Betancur $(2016,6)$ :

Es importante tener presente que para lograr un procesamiento de metales efectivo, se deben utilizar diversos productos químicos como mercurio, cianuro, ácidos concentrados y demás, lo cual altera y contamina el ciclo hidrológico afectando ecosistemas fluviales y terrestres, ya que todo ser vivo depende del agua y requiere para su sostenimiento un mínimo vital.

En cuanto al cómo se lleva a cabo la minería a cielo abierto, se debe hacer mención a la utilización de importantes maquinarias mineras (excavadoras, cintas transportadoras, entre muchas otras) y potentes insumos que son capaces de acabar con enormes superficies de tierra.

Resulta evidente que han preexistido lugares específicos en la provincia en donde se ha realizado el beneficio "que consiste en un conjunto de procesos físicos, químicos y/o metalúrgicos a los que se someten los minerales producto de la explotación, con el objeto de elevar el contenido útil o ley de los mismos [...] Las plantas de procesamiento están ubicadas en la orilla de los ríos Calera y Amarrillo. En ellas se llevan a cabo los procesos para el enriquecimiento del mineral, desde la reducción del mineral en bruto mediante molinos" (Oviedo, Moina et al. 2017, 1).

Según manifiesta el Observatorio de Conflictos Mineros en America Latina (2010, párr. 6):

El sexto Foro de los Recursos Hídricos que se reunió en Quito el 17 y 18 de junio de 2010 propuso que el Estado debe declarar en emergencia los ríos altamente contaminados por actividades mineras, industriales, uso de agrotóxicos y descargas de aguas residuales. Además, se dijo que no se debe permitir ninguna actividad minera en los nacimientos de agua, centros poblados y en las cuencas hidrográficas donde existen zonas de producción 
para el consumo local y nacional. Se debe obligar a los mineros a cumplir el título VII, referente a la preservación del medioambiente.

Por otra parte, la contaminación que ha atacado a los afluentes se ha venido desarrollando en la provincia de El Oro, "debido a los altos índices de contaminación en los cursos de agua existentes en la parte alta de la cuenca Puyango-Tumbes, que comparten Ecuador y Perú relacionados con el desarrollo de la actividad minera aurífera a pequeña escala y artesanal" (EC Ministerio del Ambiente 2011, párr. 2). Se estima que debido a ello, en el aspecto legal, las personas que han acaparado este tipo de actividades industriales han carecido de títulos mineros o concesiones otorgadas por la ARCOM, quien es el ente regulador en materia minera en el país para poder realizar ese tipo de acciones.

Según la Organizacion Mundial de la Salud (2017):

Las personas pueden estar expuestas a dos formas de mercurio en la MAPE: ${ }^{1}$ al mercurio elemental y al mercurio orgánico. El mercurio elemental se utiliza en el proceso de MAPE para formar la amalgama de oro. La forma más directa y grave de exposición es la inhalación. Cuando se calienta la amalgama de oro, se liberan altas concentraciones de vapores de mercurio elemental, por ejemplo, durante el paso de quema abierta.

López, Santos et al. $(2016,4)$ manifiestan:

Sin duda, uno de los problemas más frecuentes entre la comunidad minera aurífera es la falta de conocimiento y concienciación alrededor de la exposición a metales, en particular mercurio, entre la población. Este proceso, ligado a la ausencia de monitoreo permanente, la inexistencia de prácticas para el manejo de los desechos, y a la ausencia de políticas nacionales bien definidas para el control de estas sustancias.

\section{Sobre la minería de cobre}

La minería es una de las actividades económicas más antiguas de la humanidad. No es por nada que se clasifican las épocas prehistóricas según los minerales utilizados: edad de piedra, edad de bronce, edad de hierro. La

1. MAPE: minería aurífera artesanal y de pequeña escala. 
actividad minera mundial se desarrolla con diferentes dimensiones; estas son fundamentalmente tres: gran minería, mediana minería y pequeña minería o minería artesanal. Esta última aparece, especialmente, en los países en desarrollo (Español 2012).

El problema ecológico y, según Martínez (2016, 49), la “desestructuración territorial" son las afectaciones más recurrentes, tanto en el ámbito local como nacional e internacional, puesto que los impactos que sufren los territorios por las labores de exploración y explotación de las empresas mineras no dejan libre de daños ecológicos y socioterritoriales a ninguno de los lugares donde se llevan a cabo.

Según manifiestan Berru, Correa y Alvarado (2019, 5), "la minería contribuye en la economía de un país, pero es necesario realizarla responsablemente, procurando disminuir al máximo los daños ambientales". No solamente la extracción imprudente de oro y plata del subsuelo ecuatoriano sin los cuidados apropiados enmarcados dentro del límite de permisibilidad de la ley han dañado al medioambiente, se enfatiza en otros materiales entre los cuales se halla el cobre. Actualmente, el uso de este material se localiza en una diversidad de objetos de uso cotidiano. En efecto, la Comisión Chilena de Cobre (2017, párr. 4) expresa que "es un mineral metálico básico que no se encuentra en estado de pureza. Existen diversos tipos de yacimientos del llamado mineral rojo, que han sido clasificados de acuerdo a sus procesos geológicos".

La importancia estratégica de la industria del cobre, la gran magnitud de las inversiones realizadas y la cantidad creciente de proyectos que giran en torno a esta actividad obliga a los principales agentes a la realización de estudios que permitan a las empresas mejorar permanentemente sus sistemas productivos, comerciales y de financiación, de manera que se mantenga un adecuado equilibrio entre los aspectos coyunturales y los estructurales de la actividad (Donoso 2013).

A saber, Zorrilla (2012, párr. 17) expone:

La minería moderna utiliza y contamina enormes cantidades de agua. Para producir una tonelada de cobre puro se requiere decenas de miles de litros de este vital recurso diariamente. Por ejemplo, el proyecto minero cuprífero El Mirador, en Zamora Chinchipe, prevé utilizar cerca de 12 millones de litros por día solo para explotar y concentrar el equivalente a 200 toneladas cobre (promedio de 60.000 litros/Tn) al día. Cifras como 
esta no incluye los millones de galones de agua que tienen que ser evacuados o desviados diariamente para que no inunden el tajo y poder acceder a la mena. Mientras más profunda la mina y más pluviosidad, mayores probabilidades de encontrarse con la presencia de aguas freáticas.

\section{Minería y medioambiente en la legislación ecuatoriana}

"La industria minera ha tenido un largo y notable proceso de relación con la población, tanto por el impulso que el Estado le ha dado como por los intereses económicos alrededor de esta" (Azamar 2019, 168). A causa de la correlación existente, "Si bien en el sistema interamericano de protección de derechos humanos, y el universal no existe un compendio o un corpus iuris que determine o defina de manera expresa el medioambiente como un derecho humano autónomo" (Asprilla et al. 2019, 138).

Las jurisdicciones que emanan del poder, tanto ejecutivo como legislativo del Estado ecuatoriano, han puesto a disposición de la ciudadanía procedimientos regularizados que surten de las normas legales y sistematizan el entorno con el fin de proteger el correcto ejercicio de los derechos de las personas, tal como se encuentra expresado en la carta magna, pueden ser estas naturales, jurídicas o la propia naturaleza.

Mientras que el ejercicio de la actividad minera en el país se encuentra regulado por el Ministerio de Minería, cuyos objetivos se encuentran direccionados a priorizar la preservación de un ambiente sano, entre los cuales se cita: incrementar la productividad del sector minero; incrementar la inclusión de actores mineros en el territorio nacional; reducir el impacto ambiental y social en las actividades mineras; elevar el nivel de modernización, investigación y desarrollo tecnológico en el sector minero (EC Ministerio de Minería 2018).

La minería en sus inicios no contaba con regulaciones y base legal específica para su desarrollo, lo cual generó rechazo y desinformación en la población frente a esta actividad. Al presente y desde 2008, la minería cuenta con una remodelada ley, en la que se ha establecido una variedad de ítems que velan por una buena práctica de la actividad minera responsable, a saber en el artículo 1 "objeto de la Ley.- La presente Ley de Minería norma el ejercicio de los derechos soberanos del Estado ecuatoriano, para administrar, regular, 
controlar y gestionar el sector estratégico minero, de conformidad con los principios de sostenibilidad, precaución, prevención y eficiencia" (EC 2018, art. 1). Deviene así de los presupuestos expuestos en la misma Constitución, pero "las exigencias constitucionales indicadas pueden ser percibidas como tan estrictas que aparentemente corren el riesgo de volverse imposibles de aplicar o ser tales que impidan la explotación minera" (Vásconez Carrasco y Torres León 2018, 86).

De ello resulta necesario definir que la legislación ecuatoriana también sanciona la mala práctica de las actividades que producen afectación al suelo, aire y agua, organizado en un compendio penal punitivo en un segmento llamado "Delitos contra los recursos naturales" (EC 2014). El delito ambiental o el ilícito ambiental es el hecho típico, puesto que conlleva consecuencias legales, previstas por el derecho positivo y lesivo a los derechos del ambiente, o sea al aspecto esencial de la personalidad humana, individual y social, en relación vital con la integridad y el equilibrio del ambiente, en donde la condición de los seres vivos sea sobresaliente.

"Los delitos ambientales consideran las leyes que propenden la defensa, conservación y desarrollo armonioso del ambiente, es el marco científico-jurídico que permite limitar las situaciones irracionales que deterioran el ambiente con nefastas consecuencias para la vida del planeta" (Betancourt, López y Peñaloza 2016, 178).

De conformidad con el artículo 251 del Código Orgánico Integral Penal (EC 2014, art. 251), los delitos en contra de los recursos naturales tienen una sanción específica en cada caso. En los delitos contra el agua: "que la persona que contraviniendo la norma vigente, contamine, deseque o altere los cuerpos de agua, vertientes, fuentes, caudales ecológicos, aguas naturales, afloradas o subterráneas de las cuencas hidrográficas [...] o realicen descargas en el mar y causen daños graves, serán sancionado con una pena privativa de libertad de tres a cinco años".

De ahí que del análisis del daño ambiental existe un delito en contra del suelo, tipificado en el artículo 252 del Código Orgánico Integral Penal (EC 2014) que establece: "que la persona que contraviniendo la norma vigente, en relación con los planes de ordenamiento territorial y ambiental, cambie el uso del suelo forestal o el suelo destinado al mantenimiento y conservación de ecosistemas nativos y sus funciones ecológicas, afecte o dañe su capa 
fértil [...] provocando daños graves, será sancionada con pena privativa de libertad de tres a cinco años".

Consecuentemente, el artículo 257 del mismo cuerpo legal penal (EC 2014), manifiesta la obligación que existe de la restauración y reparación de los daños ambientales causados como: "sanciones previstas en este capítulo, se aplicara directamente con la obligación de restaurar integralmente los ecosistemas y la obligación de compensar, reparar e indemnizar a las personas y comunidades afectadas por los daños. Si el Estado asume dicha responsabilidad, a través de la Autoridad Ambiental Nacional, la repetirá contra la persona natural o jurídica que cause directa o indirectamente el daño".

\section{Análisis y resultados}

Con los objetivos planteados al inicio de la investigación y a través de los métodos utilizados, a lo largo del análisis de fuentes bibliográficas centradas en la temática se logró evidenciar el impacto ambiental que se ha generado debido a la actividad industrial minera en la provincia de El Oro, además de las ilicitudes y transgresiones que han incurrido en los cantones materia de estudio; "como consecuencia de la intervención se degradaron numerosas áreas naturales en las que, después de muchas décadas de abandono de la explotación, todavía se nota el impacto" (Ramírez y Rangel 2019, 673). A causa de esto, la principal víctima resultó ser el medioambiente.

Los recursos minerales han sido esenciales desde el origen de las civilizaciones, de manera que hoy en día cualquier sociedad moderna hace un uso extensivo e intensivo de ellos para el desarrollo de su actividad económica y para impulsar la consabida calidad de vida, a través de un uso más amplio de productos y enseres fabricados con diferentes materias primas (Fueyó 2019). Respectivamente, "el desarrollo de actividades mineras bajo una responsabilidad social y ambiental es un reto ambiental, social, cultural, económico y político para el gobierno" (Pinilla y Santos 2014, 473).

En fin, de acuerdo con el Instituto Nacional de Estadística y Censos (2010, 283): 
La provincia de El Oro acoge el mayor número de personas que trabajan en la actividad de explotación de minas y canteras en el Ecuador, siendo el principal cantón Portovelo con 1.694 personas y 1.247 en Zaruma, cantones que poseen la mayor concentración de individuos dedicados a la minería, Machala posee 639, Piñas 612, Santa Rosa 499, Pasaje 343, Atahualpa 330.

Como se puede observar, la actividad minera concentra gran parte de la economía de la provincia de El Oro, permitiendo que sus habitantes lleven el sustento diario a sus hogares; por lo tanto, es una de las razones por las que esta actividad se debe desarrollar responsablemente, en armonía con el medioambiente y la naturaleza.

\section{Materiales y métodos}

El presente trabajo es una investigación de tipo descriptiva y cualitativa en la obtención de resultados a través de la observación. El diseño de investigación cualitativo escogido permitió establecer una diferencia entre los datos recopilados por las fuentes bibliográficas y los resultados obtenidos por los investigadores a través de la observación.

Los métodos utilizados son el inductivo y el comparativo, con los cuales se ha podido desglosar cada uno de los aspectos de la minería ilegal y su contaminación y se ha realizado una comparación con otros casos e investigaciones. La técnica utilizada especialmente para este trabajo es la observación.

\section{Discusión y conclusiones}

Para finalizar, la minería que se ha ejecutado en todas sus escalas debería contar con un sistema de defensa social y una exhaustiva vigilancia por los diversos órganos del poder público que se relacionan con los productos de la tierra ecuatoriana o sus yacimientos minerales; en consecuencia, correspondería realizar un trabajo en conjunto y no por separado, como se ha venido procurando. 
El Ecuador ha descuidado sus áreas económicas, sociales y ambientales en el tema de la minería, causando que la falta de inversión ocasione pérdidas y daños en el medioambiente; por lo tanto, es aconsejable trabajar en políticas públicas que permitan aprovechar los recursos naturales sin afectar la naturaleza. Si se continua de esta manera será imposible mermar el impacto ambiental que la contaminación y la extracción de recursos está causando, que además de ser perjudicial para la Pacha Mama, es vital para la existencia de todo ser humano. Esto no significa que hay que acabar con esta actividad industrial porque es vital para el desarrollo continuo del país. Decir no a la minería irresponsable es un compromiso de todos.

\section{Referencias}

Alcívar, Edna. 2015. "Manejo de los desechos sólidos en la provincia de El Oro y su impacto ambiental en los ecosistemas". Tesis de grado, Universidad Técnica de Machala, Machala. Accedido enero de 2015. http://repositorio.utmachala.edu.ec/handle/48000/2949.

Alvarado, Julio. 2017. "Impactos económicos y sociales de las políticas nacionales mineras en Ecuador (2000-2006)". Revista de Ciencias Sociales 23 (4): 53-64. https://www.redalyc. org/articulo.oa?id=28055641005.

Asprilla, Edwin, Hames Lozano, Rafael Bechara y Ricardo Ledezma. 2019. "El medio ambiente como víctima del conflicto armado en el departamento del Chocó". Pensamiento Americano 12 (23): 127-144. https://doi.org/10.21803/pensam.v12i22.251.

Azamar, Aleida. 2019. "Minería y Estado: una relación permisiva". Pós Ciencias Sociais 16 (32): 167-187. http://dx.doi.org/10.18764/2236-9473.v16n32p167-187.

Banco Central del Ecuador. 2017. "Reporte de minería”. Dirección Nacional de Síntesis Macroeconómica. Accedido 14 de abril del 2020. https://bit.ly/2VdBhfh.

Berru, Juan, Henry Correa y Laura Alvarado. 2019. "Alcalinidad del agua-río Tenguel. Asociación Comunitaria Minera Corralitos en el área de explotación. Caso de estudio Ecuador". Revista Desarrollo Local Sostenible 12 (34): 23-28. https://www.eumed.net/rev/ delos/33/index.html.

Betancourt, Yusmeny, Emma López y Anival Peñaloza. 2016. "Daños y delitos ambientales como conceptos discernibles en la enseñanza de la química del Instituto pedagógico de Caracas. Estudio preliminar desde la perspectiva estudiantil". Revista de Investigación 40 (8): 176-201. http://www.scielo.org.ve/pdf/ri/v40n88/art10.pdf.

Bouzas, Alfonso, Juan José Cirión, Perla Gómez, Aleida Hernández, Rafael Lara, Rosalio López, Alfonso Ochoa, Germán Reyes y Laura Suárez. 2007. Epistemología y Derecho. Ciudad de México: Universidad Nacional Autónoma de México, IIEc-UNAM. 
Bouzas, José. 2015. Las nuevas condiciones del trabajo en el contexto de la globalización económica. ¿Hacia un nuevo derecho del trabajo? Ciudad de México: Universidad Nacional Autónoma de México / Consejo Nacional de Ciencia y Tecnología / Instituto de Investigaciones Económicas. ISBN 978-607-02-7252-3.

Castro, Daniela. 2019. "Situación actual minería en Ecuador”. Tesis de pregrado, Universidad de las Américas, Quito. https://bit.ly/2NhPSBY.

Comision Chilena de Cobre. 2017. Tendencias de usos y demanda de productos de cobre. Santiago: Ministerio de Minería. https://bit.ly/2YV03li.

Cueto, Gerardo. 2016. "Nuevos usos turísticos para el patrimonio minero en España". PASOS: Revista de Turismo y Patrimonio Cultural 5 (12): 1013-1026. https://doi.org/10.25145/j. pasos.2016.14.065.

Donoso, Manuel. 2013. "El mercado del cobre a nivel mundial: evolución, riesgos, características y potencialidades futuras". Ingeniare. Rev. chil. ing. 21 (2): 248-261. http://dx.doi. org/10.4067/S0718-33052013000200008.

EC. 2014. Código Orgánico Integral Penal. Registro Oficial 180, Suplemento, 10 de febrero.

---. 2018. Ley de Minería. Registro Oficial, Suplemento 517, 29 de enero de 2009.

EC Agencia de Regulación y Control Minero. 2015. Proyecto de seguimiento, control y evaluación de labores mineras en el distrito Zaruma-Portovelo. Quito: ARCOM. https://bit. ly/2AKzg3a.

EC Instituto Nacional de Estadística y Censos (INEC). 2010. "Número de personas dedicadas a la explotación de minas y canteras por cantones en la provincia de El Oro". Quito: INEC. https://bit.ly/3diW0V3.

EC Ministerio de Minería. 2016. Plan Nacional de Desarrollo del Sector Minero. Accedido 4 de junio de 2020. https://bit.ly/3diW0V.

EC Ministerio del Ambiente. 2011. Programa de reparacion ambiental y social. 3 de marzo. Accedido 4 de junio de 2020. https://bit.ly/3fEsE5a.

Español, Santiago. 2012. "Contaminación con mercurio por la actividad minera". Biomédica 32 (3): 20-29. https://doi.org/10.7705/biomedica.v32i3.1437.

Fueyo Editores. 2019. "Nuevo manual sobre los minerales críticos y estratégicos en la nueva economía". Rocas y minerales: técnicas y procesos de minas y canteras 565: 12-13. https: //bit.ly/2V4h4s1.

González, Víctor, Sonia Valle, Mauro Nirchio, Jesús Olivero, Lesly Tejeda, Juan Valdemar, Fredis Pesantes y Katherine González. 2018. "Evaluación del riesgo de contaminación por metales pesados $(\mathrm{Hg}$ y $\mathrm{Pb}$ ) en sedimentos marinos del estero Huaylá, Puerto Bolívar, Ecuador". Revista del Instituto de Investigación de la Facultad de Ingeniería Geológica, Minera, Metalúrgica y Geográfica 21 (41): 75-82. https://doi.org/10.15381/iigeo. v21i41.14995.

Henry, Glynn, y Gary Heinke. 1999. Ingeniería ambiental. Ciudad de México: Prentice Hall Hispanoamericana. 


\section{Wilson Vilela-Pincay, Marbelle Espinosa-Encarnación y Ana Bravo-González}

López, Marcelo, Jovanny Santos, César Quezada, Marisela Segura y Johnny Pérez. 2016. "Actividad minera y su impacto en la salud humana". UNEMI 9 (17): 92-100. https:// dialnet.unirioja.es/descarga/articulo/5556797.pdf.

Martínez, Hirineo. 2016. "Concesiones, explotación minera y conflicto en la frontera Jalisco-Colima". Espiral (Universidad de Guadalajara) 23 (67): 45-90. https://bit.ly/2V2tRvg.

Moussa, Nicole. 1999. "La demanda del cobre". En El desarrollo de la mineria del cobre en la segunda mitad del siglo XX, editado por Nicole Moussa, 53-63. Santiago de Chile: Naciones Unidas. https://bit.ly/3fIN39c.

Observatorio de Conflictos Mineros en América Latina. 2010. Emergencia: Ríos Calera y Amarillo altamente contaminados por actividad minera. Accedido 4 de junio de 2020. https://bit.ly/2ATIISR.

Orellana, Isabel, Laurence Briére y Felipe Rodríguez, 2020 “"La resistencia social en contexto de conflicto socioecológico: un crisol de desarrollo de las dimensiones crítica y política de la educación ambiental". Ambiente \& Educación 25 (1): 13-45. 10.14295/ambeduc. v25i1.11117.

Organización Mundial de la Salud. 2017. "Documento técnico No. 1: riesgos para la salud relacionados con el trabajo y el medio ambiente asociados a la extracción de oro artesanal o a pequeña escala". Accedido 12 de junio de 2020. https://apps.who.int/iris.

Oviedo, Rodrigo, Emy Moina, Jaime Naranjo y Milton Barcos. 2017. "Contaminación por metales pesados en el sur del Ecuador asociada a la actividad minera". Revista Bionatura 2 (4): 437-441. 10.21931/RB/2017.02.04.5.

Pásara, Luis. 1974. "Algunas hipótesis sobre las reformas de la minería de cobre”. Derecho PUCP: Revista de la Facultad de Derecho 32: 218-228. http://revistas.pucp.edu.pe/index. $\mathrm{php} /$ derechopucp/article/view/6149.

Paucar, Elena. 2017. "Zaruma se hunde por la minería". El Comercio. 9 de enero. Accedido 15 de junio de 2020. https://bit.ly/2NJz4nD.

Pérez, Margarita, y Angie Betancur. 2016. "Impactos ocasionados por el desarrollo de la actividad minera al entorno natural y situación actual de Colombia”. Sociedad y Ambiente 4 (10): 95-112. 10.31840/sya.v0i10.1654.

Pinilla Pedraza, Claudia, y Clara Santos Morán. 2014. "La minería ilegal arrasando las entrañas de la Tierra". Mundo Amazónico 5 (1): 455-475. 10.15446/ma.v5.45755.

Ramírez, Giovanny, y Orlando Ranchel. 2019. "Sucesión vegetal en áreas de minería a cielo abierto en el bosque pluvial tropical del departamento del Chocó, Colombia". Rev. Acad. Colomb. Cienc. Exact. 43 (169): 673-688. http://dx.doi.org/10.18257/raccefyn.896.

Ríos, Tatiana. 2015. VisitaElOro.com. Accedido 10 de junio de 2020. https://bit.ly/3enge1k.

Román, Jorge. 2020. "Zaruma se hunde por la minería clandestina". eluniverso.com. 3 de febrero. Accedido 10 de junio. https://bit.ly/3fIpn4A.

Salazar, Diego, Victoria Castro, Jaie Michelow, Hernán Salinas, Valentina Figueroa y Mille Benoit. 2010. "Minería y metalurgia en la costa Arreica de la región de Antofagasta, norte 
de Chile”. Boletín del Museo Chileno de Arte Precolombino 15 (1): 9-23. http://dx.doi. org/10.4067/S0718-68942010000100002.

Sánchez, Enrique, y María Ortiz. 2014. "Escenarios ambientales y sociales de la minería a cielo abierto". Inventio, la génesis de la cultura universitaria en Morelos 20: 27-34. https:// bit.ly/2CrwRuB.

Sánchez, Luis, Esbe Leifsen y Ana Verdú. 2017. "Minería a gran escala en Ecuador: conflicto, resistencia y etnicidad". Revista de Antropología Iberoamerican 12 (2): 169-192. 10.11156/aibr.120205.

Sánchez, Luis, María Espinosa y María Eguiguren. 2016. "Percepción de conflictos socioambientales en zonas mineras: el caso del proyecto mirador en Ecuador". Ambiente y Sociedade 19 (2): 23-42. https://bit.ly/2YgnUwx.

Vargas, Juan, y Diego Manrique. 2015. “Análisis económico de la minería de carbón y oro 2007-2010 en Colombia”. Contexto 4: 59-70. https://bit.ly/2Ncm3CN.

Vásconez Carrasco, Marcelo, y Leonardo Torres León. 2018. "Minería en el Ecuador: sostenibilidad y licitud". Estudios del Desarrollo Social: Cuba y América Latina 6 (2): 83-103. https://bit.ly/2YSnSu3.

Vázquez, Alba, y Enrique Valdez. 1994. Impacto ambiental. Ciudad de México: Facultad de Ingeniería de la Universidad Nacional Autónoma de México. https://bit.ly/3deGCc5.

Zorrilla, Carlos. 2012. "La minería de cobre y sus inpactos en el Ecuador". La Linea de Fuego. Accedido 15 de junio de 2020. https://bit.ly/2YSHEp0. 